\title{
Exercise and Mitochondrial Remodeling in Skeletal Muscle in Type 2 Diabetes
}

\author{
Hojun Lee ${ }^{1,2}$, Wook Song ${ }^{3,4, *}$ \\ ${ }^{1}$ Department of Rehabilitation Medicine, Seoul National University Bundang Hospital, Seongnam; ${ }^{2}$ Department of Sports and Health Science, Kyungsung University, \\ Busan; ${ }^{3}$ Health and Exercise Science Laboratory, Institute of Sport Science and ${ }^{4}$ Institute on Aging, Seoul National University, Seoul, Korea
}

Exercise is regarded as a potent stimulus in modulation of glucose utility and mitochondrial adaptations in skeletal muscle, leading to enhanced metabolic health. As mitochondria play a crucial role in sustaining metabolic homeostasis, and disturbances in mitochondrial function are highly linked with development of metabolic diseases, a comprehensive understanding of exercise-mediated mitochondrial remodeling under the pathophysiological condition of type 2 diabetes is warranted to develop an efficient therapeutic strategy. Although it is evident that the primary etiology of type 2 diabetes is insulin resistance, there is accumulating evidence linking abnormal mitochondrial functional and morphological properties to development of type 2 diabetes. Despite this, the precise molecular and cellular events that underline these phenomena remain uncertain. Mitochondria are highly dynamic subcellular organelles that can change mass and shape as necessary via coordinated processes such as mitochondrial fusion, fission, and biogenesis. Mitochondrial fusion is controlled by proteins, including mitofusin-1, mitofusin-2, and optic atrophy protein 1, while the fission process is mainly modulated by control of fission protein 1 and dynamin-related protein 1. Peroxisome proliferator-activated receptor gamma coactivator1 a acts as a master controller of mitochondrial biogenesis. The present review's primary aims were to briefly discuss the cellular mechanisms of muscle fiber type-dependent glucose uptake and to highlight emerging evidence linking disturbances in mitochondrial dynamics to development of insulin resistance and type 2 diabetes. The potential for exercise to normalize type 2 diabetes-induced aberrant mitochondrial integrity is also addressed.

Key words: Mitochondrial dynamics, Mitochondrial biogenesis, Exercise, Type 2 diabetes mellitus
Received August 3, 2018

Reviewed August 20, 2018

Accepted September 3, 2018

${ }^{*}$ Corresponding author

Wook Song

(i)

https://orcid.org/0000-0002-8825-6259

Health and Exercise Science Laboratory, Institute of Sport Science, Seoul National University, 1 Gwanak-ro, Gwanak-gu, Seoul 08826, Korea

Tel: +82-2-880-7791

Fax: +82-2-872-2867

E-mail: songw3@snu.ac.kr

\section{INTRODUCTION}

Although direct causality between mitochondria and insulin resistance is still under investigation, a wide spectrum of evidence indicates that mitochondrial dysfunction is associated with increased insulin resistance in skeletal muscle. ${ }^{1,2}$ Muscle insulin resistance is the manifestation of type 2 diabetes, as skeletal muscle is the largest human organ and is involved in approximately one third of whole body energy metabolism at rest and up to $90 \%$ during active exercise. ${ }^{3}$ As the mitochondrion is an energy powerhouse responsible for complete oxidation of glucose- and fat-derived metabolites to generate adenosine triphosphate $(\mathrm{ATP})^{4}$, skeletal muscle bioenergetics is considered to be a major factor in metabolic health. Skeletal muscle mitochondria are highly interconnected as a form of reticulum that promotes the relocation of substrates and metabolites to bioenergetically active areas within mitochondria. ${ }^{5}$ Thus, it is believed that, along with increases in mitochondrial mass, changes in mitochondrial morphology via fusion and fission are significantly related with development of metabolism-related diseases.

Exercise is a potent and nonpharmaceutical intervention for management and treatment of a wide spectrum of lifestyle-related diseases. ${ }^{6,7}$ While the therapeutic effects of exercise are unequivo-

Copyright (C) 2018 Korean Society for the Study of Obesity

(a) This is an Open Access article distributed under the terms of the Creative Commons Attribution Non-Commercial License (http://creativecommons.org/licenses/by-nc/4.0/) which permits unrestricted non-commercial use, distribution, and reproduction in any medium, provided the original work is properly cited. 
$\mathrm{cal}$, the exercise-mediated molecular responses that modulate mitochondrial dynamics under type 2 diabetes remain relatively less known. In this miniature review, we will briefly introduce how plasma glucose is absorbed into skeletal muscle. We will also emphasize major molecular mechanisms between mitochondrial biogenesis, fusion, and fission, with respect to how mitochondria dynamics are dysregulated under type 2 diabetes and how exercise may orchestrate the mitochondrial reticulum.

\section{INSULIN-DEPENDENT GLUCOSE UPTAKE IN SKELETAL MUSCLE}

It has been well documented that pancreas-secreted insulin initiates the transportation of plasma glucose into myofibers via a series of molecular signaling cascades. ${ }^{8}$ This cellular process is accomplished by relocating glucose transporter type 4 (GLUT4) to the plasma membrane (Fig. 1). Briefly, upon insulin binding to the insulin receptor located on the periphery of skeletal muscle cells, the insulin receptor is autophosphorylated to induce subsequent activation of insulin receptor substrate 1 (IRS1) and PI3 kinase. ${ }^{8}$ This

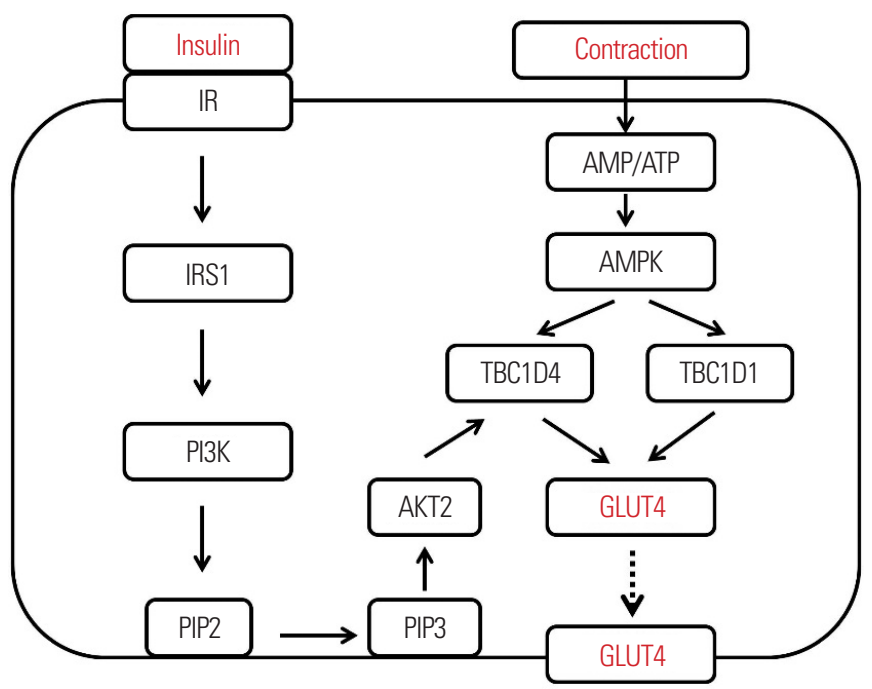

Figure 1. Independent modulation of insulin- and muscle contraction-induced glucose uptake into myofibers. The line arrows indicate activation of a series of signaling pathways. The dotted arrow indicates translocalization of GLUT4. IR, insulin receptor; IRS1, insulin receptor substrate 1; PI3K, phosphatidylinositol-4,5-bisphosphate 3-kinase; PIP2, phosphatidylinositol 4,5-bisphosphate; AMP, 5' adenosine monophosphate; ATP, adenosine triphosphate; AMPK, AMP-activated protein kinase; TBC1D4 and 1, tre-2/USP6, BUB2, cdc16 domain family members 4 and 1; AKT2, protein kinase B2; GLUT4, glucose transporter type 4; PIP3, phosphatidylinositol 3,4,5-bisphosphate. promotes the conversion of phosphatidylinositol 4,5-bisphosphate to phosphatidylinositol 3,4,5-triphosphate at the plasma membrane, which ultimately induces phosphorylation and conformational changes of protein kinase B2 (AKT2; a master controller of GLUT4). ${ }^{9}$ Once AKT2 is activated, cytosolic GLUT4 is translocalized to the plasma membrane with the help of signal-relaying molecules to induce intake of plasma glucose into myofibers. ${ }^{10,11}$

\section{MUSCLE CONTRACTION-INDUCED GLUCOSE UPTAKE IN SKELETAL MUSCLE}

Skeletal muscle contraction has been identified as a potent independent stimulus to induce glucose uptake into myofibers, which is also mainly modulated by the expression and translocation of GLUT4. ${ }^{12}$ Interestingly, although it is evident that insulin sensitivity is improved by exercise and muscle contraction ${ }^{13}$, the major mechanism(s) by which muscle contraction increases the rate of glucose uptake is somewhat independent of insulin action. This notion has been documented by several lines of evidence indicating that deletion of major insulin signaling markers (IRS1 and AKT2) does not diminish muscle contraction-induced glucose uptake $\mathrm{e}^{14,15}$ and insulin combined with muscle contraction stimulates glucose uptake into myofibers synergistically. ${ }^{16,17}$ There are potent molecular markers initiating insulin-independent glucose uptake in skeletal muscle. Among them, 5' adenosine monophosphate-activated protein kinase (AMPK) has garnered significant attention in the past few decades. AMPK consists of an $\alpha$ catalytic subunit and two types of regulatory subunits $(\beta$ and $\gamma) .{ }^{18}$ All three types of subunits work in coordination for maximal activation of the enzyme. ${ }^{19}$ The enzymatic activation of this kinase is primarily induced by energy-demanding conditions such as skeletal muscle contraction and orchestrates an energy-related network via minimizing the ATPconsuming pathway and maximizing the ATP-generating pathway (i.e., glucose uptake, mitochondrial biogenesis, and remodeling). ${ }^{8}$ In regard to glucose uptake, the critical downstream molecules of AMPK are tre-2/USP6, BUB2, cdc16 domain family members 1 and 4 (TBC1D1 and TBC1D4). ${ }^{20,21}$ AMPK-induced phosphorylation of specific sites of TBC1D1 and TBC1D4 leads to translocation of glucose transporter (GLUT4) to the myofiber membrane. ${ }^{22,23}$ Once GLUT4 is incorporated into the membrane, plasma glucose 
is funneled into myofiber through GLUT4 by passive diffusion. When myofibers are under an oxygen-rich condition, glucose-derived metabolites are further processed in mitochondria for complete oxidation. ${ }^{24}$ Therefore, the enhanced quality of mitochondria is considered to be linked with muscle glucose uptake and metabolism in a coordinated manner.

\section{SKELETAL MUSCLE FIBER TYPE AND GLUCOSE UTILIZATION}

Although skeletal muscle, the largest organ of the body, is considered to be a significant contributor to modulation of glucose uptake and metabolism ${ }^{25}$, individual muscles may contribute differently based on fiber type composition. ${ }^{26}$ Type I myofibers embedded with abundant mitochondria have a higher glucose-processing capacity, mainly due to a greater mitochondrial oxidative capacity for substrate utilization. ${ }^{27}$ On the contrary, type II muscle fibers embedded with less mitochondria are considered to be less insulinsensitive and contribute less to substrate oxidation. ${ }^{28}$ This notion has been further supported by a human biopsy study of greater insulin responsiveness in slow-twitch fiber. ${ }^{29}$ Aerobic exercise has been consistently reported as a potential strategy to increase mitochondrial oxidative capacity in skeletal muscle and induce the transition of myofiber to more oxidative traits. ${ }^{30}$ In that regard, a physically active lifestyle and regular aerobic exercise are warranted to prevent or manage type 2 diabetes.

\section{MITOCHONDRIAL BIOGENESIS AND TYPE 2 DIABETES}

Skeletal muscle is the dominant site of both insulin-dependent and -independent glucose utilization in the body. While glucose could be metabolized in cytoplasm as a rapid form of glycolysis, a majority of glucose-derived metabolites is funneled into the mitochondrial matrix for oxidative metabolism through a series of enzymatic processes to generate ATP. ${ }^{31}$ Therefore, disrupted mitochondrial biogenesis is considered to diminish the ability of skeletal muscle to oxidize glucose-derived substrates and can have negative consequences on glucose uptake in skeletal muscle. ${ }^{32}$

A wide variety of signaling molecules serve as a platform for or- chestrating the coordinated assembly of mitochondrial reticulum. In particular, the peroxisome proliferator-activated receptor-gamma coactivator-1 (PGC-1) family has garnered significant attention. Among them, PGC-1 $\alpha$ has been extensively studied due to its versatile and dynamic ability to induce the expression of mitochondrial biogenesis- and substrate oxidation-related genes in both nuclear and mitochondrial genomes. ${ }^{33}$ This complex process is initiated via deacetylation of PGC-1 $\alpha$, followed by its individual binding to multiple transcription factors. When combined with certain nuclear transcription factors (i.e., NRF-1 and NRF-2), PGC-1 $\alpha$ guides them to translocate from the cytoplasmic region to specific promoter sites of nuclear DNA, allowing for the expression of numerous mitochondrial components. ${ }^{34}$ Although a vast majority of mitochondrial genes $(\sim 1,300)$ are transcriptionally expressed in nuclear DNA, a separate set of 13 mitochondrial genes is encoded in a circular form of the mitochondrial genome. ${ }^{35}$ When PGC- $1 \alpha$ is connected to mitochondrial transcription factor A in cytoplasm, they localize to mitochondrial DNA, increasing the number of mitochondrial DNA copies. ${ }^{36}$ Therefore, PGC- $1 \alpha$ is considered as a master regulator of mitochondrial biogenesis.

There are a series of studies reporting that deficiencies in mitochondrial content, oxidative phosphorylation, and substrate oxidation are observed in skeletal muscles of subjects with types 2 diabetes and metabolic syndrome. ${ }^{37-39}$ The reduced mitochondrial contents and function are retained even following biopsy-derived cultures of myocytes obtained from individuals with type 2 diabetes. ${ }^{40}$ These changes were linked with reduced mitochondrial mass and density, suggesting that PGC-1 $\alpha$ may be an early biomarker in metabolic diseases. ${ }^{34,41}$ This notion is further supported by a study indicating that rodents abundant with PGC-1 1 tend to have not only higher mitochondrial capacity, but also greater glucose utilization ${ }^{41}$, suggesting the importance and implication of exercise-induced PGC-1 $\propto$ in skeletal muscles.

\section{MITOCHONDRIAL DYNAMICS AND TYPE 2 DIABETES}

Mitochondrion, a cellular energy power house, changes its shape, size, and location to adapt to fluctuating energetic demands. ${ }^{42}$ These changes are accomplished through the coordinated cycles of 


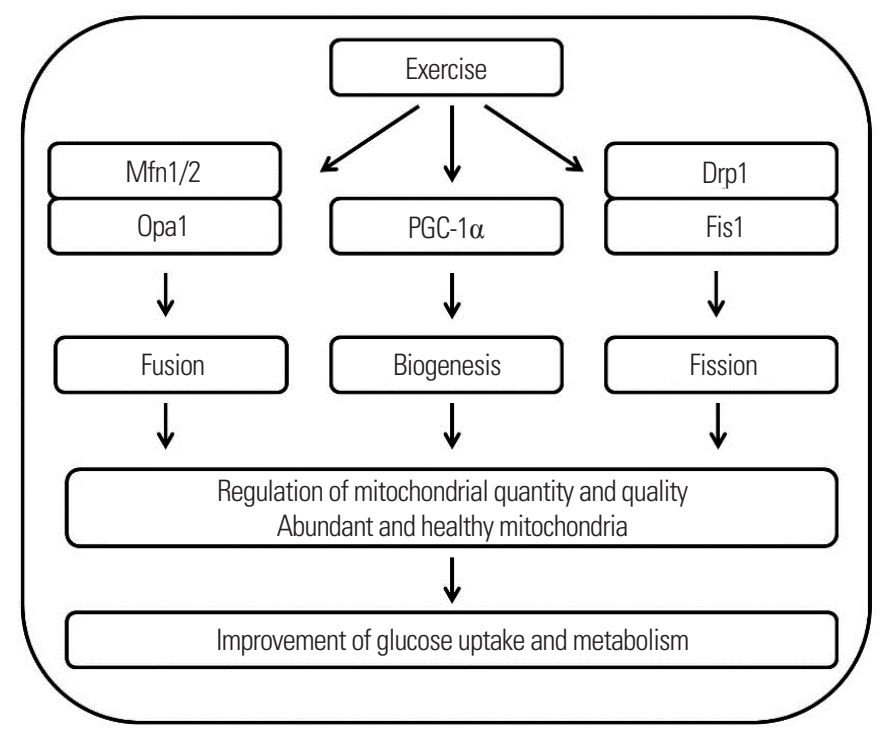

Figure 2. Schematic diagram of exercise-mediated mitochondrial quality and quantity control in skeletal muscle and its implications in the improvement of glucose uptake and metabolism. The arrows indicate molecular and physiological processes. Mfn, mitofusin; Opa1, optic atrophy protein 1; PGC-1 $\alpha$, proliferator-activated receptor-gamma coactivator-1 $\alpha$; Drp1, dynamin-related protein 1; Fis1, fission protein 1 .

mitochondrial biogenesis, fission, and fusion. ${ }^{43}$ Mitochondrial biogenesis is defined as the addition of new mitochondria. Mitochondrial fusion produces large interconnected reticulum of mitochondria, whereas small fragmented mitochondria are created via a process called fission. Balanced coordination of these complex networks is essential for maximizing mitochondrial efficiency, suggesting that mitochondrial quality control is as important as mitochondrial quantity for substrate oxidation (Fig. 2). Briefly, mitochondrial fusion is mainly modulated by a group of GTPases identified as mitofusins (Mfns) and optic atrophy protein 1 (Opa1). ${ }^{44} \mathrm{Mfn} 1 / 2$ are required to fuse the outer mitochondrial membrane, while Opal induces fusion of the mitochondrial inner membrane. ${ }^{44} \mathrm{Dy}$ namin-related protein 1 and fission protein 1 (Fis1) are recruited to the outer mitochondrial membrane to induce mitochondrial division. ${ }^{45}$ Cellular events of fission and fusion are tightly monitored and modulated under normal physiological conditions, as imbalances in mitochondrial dynamics lead to the development of various types of diseases including metabolic diseases. ${ }^{46,47}$ It has been reported that the morphological structure of mitochondria is different in patients with type 2 diabetes versus in healthy individuals. ${ }^{48}$ This suggests that aberrant mitochondrial morphology is re- lated with key remodeling proteins. In line with this idea, the protein expression of mitochondrial fusion (Mfn2 and Opa1) has been reported to be reduced in skeletal muscles of patients with type 2 diabetes. ${ }^{48,49}$ Additionally, animal models of dysfunctional $\mathrm{Mfn} 2$ demonstrate reduced substrate metabolism ${ }^{50}$, whereas overexpression of Mfn2 and Opa1 restores mitochondrial respiration efficiency, glucose oxidation, and insulin resistance. ${ }^{51,52}$ Although it seems to be evident that aberrant mitochondrial dynamics are associated with glucose metabolism and insulin resistance, several studies have shown that aberrant mitochondrial abnormity is not observed in skeletal muscles with type 2 diabetes ${ }^{53,54}$, inducing an active discussion of whether mitochondrial abnormality is a consequence or cause of type 2 diabetes. Therefore, future studies with an integrative approach (cell to human) are warranted to delineate the specific mechanisms of mitochondrial dynamics in type 2 diabetes.

\section{TARGETING MITOCHONDRIAL DYNAMICS THROUGH EXERCISE}

Although exercise-induced mitochondrial biogenesis has been well documented, the effects of exercise on mitochondrial dynamics in skeletal muscle have been less extensively explored. Several recent studies have indicated that skeletal muscle contraction appears to have a capacity to modulate both mitochondrial biogenesis and dynamics in a coordinated manner, suggesting that exercise and physical activity modulate not only mitochondrial quantity, but also quality in skeletal muscle in a synergistic manner. For example, high-intensity aerobic exercise has been demonstrated to induce the protein expression of mitochondrial fusion (Mfn1) and fission (Fis1). ${ }^{55}$ In agreement with this, a single bout of aerobic exercise increased the messenger RNA expression of $\mathrm{Mfn} 1$ and $\mathrm{Mfn} 2$ in the skeletal muscles of both rodents and humans. ${ }^{56,57}$ However, the specific upstream mechanism regulating these processes during exercise remains largely unknown. In line with this, although it was tested in an in vitro condition, a novel study showed that mitochondrial fusion is regulated by PGC- $1 a$ via estrogen-related receptor $\alpha .{ }^{56}$ Given that exercise-induced expression of PGC- $1 \alpha$ precedes induction of Mfn1 and $\mathrm{Mfn} 2^{56}$, it is highly likely that not only might mitochondrial biogenesis, but also the dynamics of mito- 
chondrial fusion and fission be regulated by PGC- $1 \alpha .^{58}$ Based on previous research indicating that aberrant mitochondrial integrity is associated with development of type 2 diabetes and that increased expression of PGC-1 $\alpha$ in skeletal muscle is a central dogma in exercise physiology, identifying the optimal exercise volume and intensity to maximize induction of PGC-1 $\alpha$ in skeletal muscle is warranted to provide a potential nonpharmaceutical strategy for patients with type 2 diabetes.

\section{CONCLUSION}

The regulation of glucose uptake and metabolism in skeletal muscle is significantly controlled in a mitochondrial health-dependent manner, and aberrant mitochondrial functional properties in skeletal muscle are linked with development of type 2 diabetes. The quantity and quality of mitochondria are modulated via a series of coordinated cellular processes of mitochondrial biogenesis, fusion, and fission. Multiple studies discussed in this review suggest that the major signaling proteins controlling mitochondrial remodeling are dysregulated in the skeletal muscle of type 2 diabetes, and that exercise has a great potential to regulate not only mitochondrial biogenesis, but also dynamics, improving the overall quality of these organelles in diabetic skeletal muscle. While the therapeutic effects of exercise are unequivocal, the exercise mode-, intensity-, and volume-mediated molecular responses modulating mitochondrial dynamics in patients with type 2 diabetes remain relatively less identified. In that regard, it will be crucial that future research be devoted to elucidating the interplay between specific exercise protocols, mitochondrial dynamics, and type 2 diabetes to provide a stepping stone for development of a novel therapeutic exercise strategy.

\section{CONFLICTS OF INTEREST}

The authors declare no conflict of interest.

\section{ACKNOWLEDGMENTS}

This work was supported by the National Research Foundation of Korea funded by the Ministry of Science, ICT and Future Plan- ning (NRF-2013M3A9B6046417, Korea Mouse Phenotyping Project NRF-2013M3A9D5072550, 2013M3A9D5072560, 2017M3A9D5A01052447, and MEST 2011-030135).

\section{REFERENCES}

1. Martin SD, McGee SL. The role of mitochondria in the aetiology of insulin resistance and type 2 diabetes. Biochim Biophys Acta 2014;1840:1303-12.

2. Szendroedi J, Phielix E, Roden M. The role of mitochondria in insulin resistance and type 2 diabetes mellitus. Nat Rev Endocrinol 2011;8:92-103.

3. Zurlo F, Larson K, Bogardus C, Ravussin E. Skeletal muscle metabolism is a major determinant of resting energy expenditure. J Clin Invest 1990;86:1423-7.

4. Szendroedi J, Schmid AI, Chmelik M, Toth C, Brehm A, Krssak M, et al. Muscle mitochondrial ATP synthesis and glucose transport/phosphorylation in type 2 diabetes. PLoS Med 2007; 4:e154.

5. Glancy B, Hartnell LM, Malide D, Yu ZX, Combs CA, Connelly PS, et al. Mitochondrial reticulum for cellular energy distribution in muscle. Nature 2015;523:617-20.

6. Colberg SR, Sigal RJ, Fernhall B, Regensteiner JG, Blissmer BJ, Rubin RR, et al. Exercise and type 2 diabetes: the American College of Sports Medicine and the American Diabetes Association: joint position statement executive summary. Diabetes Care 2010;33:2692-6.

7. Sigal RJ, Kenny GP, Wasserman DH, Castaneda-Sceppa C, White RD. Physical activity/exercise and type 2 diabetes: a consensus statement from the American Diabetes Association. Diabetes Care 2006;29:1433-8.

8. O'Neill HM. AMPK and exercise: glucose uptake and insulin sensitivity. Diabetes Metab J 2013;37:1-21.

9. Alessi DR, Andjelkovic M, Caudwell B, Cron P, Morrice N, Cohen P, et al. Mechanism of activation of protein kinase B by insulin and IGF-1. EMBO J 1996;15:6541-51.

10. Peck GR, Chavez JA, Roach WG, Budnik BA, Lane WS, Karlsson $\mathrm{HK}$, et al. Insulin-stimulated phosphorylation of the Rab GTPase-activating protein TBC1D1 regulates GLUT4 translocation. J Biol Chem 2009;284:30016-23. 
11. Sano H, Kane S, Sano E, Mîinea CP, Asara JM, Lane WS, et al. Insulin-stimulated phosphorylation of a Rab GTPase-activating protein regulates GLUT4 translocation. J Biol Chem 2003;278:14599-602.

12. Richter EA, Hargreaves M. Exercise, GLUT4, and skeletal muscle glucose uptake. Physiol Rev 2013;93:993-1017.

13. Holloszy JO. Exercise-induced increase in muscle insulin sensitivity. J Appl Physiol (1985) 2005;99:338-43.

14. Dumke CL, Wetter AC, Arias EB, Kahn CR, Cartee GD. Absence of insulin receptor substrate-1 expression does not alter GLUT1 or GLUT4 abundance or contraction-stimulated glucose uptake by mouse skeletal muscle. Horm Metab Res 2001; 33:696-700.

15. Sakamoto K, Arnolds DE, Fujii N, Kramer HF, Hirshman MF, Goodyear LJ. Role of Akt2 in contraction-stimulated cell signaling and glucose uptake in skeletal muscle. Am J Physiol Endocrinol Metab 2006;291:E1031-7.

16. King PA, Betts JJ, Horton ED, Horton ES. Exercise, unlike insulin, promotes glucose transporter translocation in obese Zucker rat muscle. Am J Physiol 1993;265(2 Pt 2):R447-52.

17. Brozinick JT Jr, Etgen GJ Jr, Yaspelkis BB 3rd, Ivy JL. Contraction-activated glucose uptake is normal in insulin-resistant muscle of the obese Zucker rat. J Appl Physiol (1985) 1992; 73:382-7.

18. Hardie DG, Ross FA, Hawley SA. AMPK: a nutrient and energy sensor that maintains energy homeostasis. Nat Rev Mol Cell Biol 2012;13:251-62.

19. Dyck JR, Gao G, Widmer J, Stapleton D, Fernandez CS, Kemp $\mathrm{BE}$, et al. Regulation of 5'AMP-activated protein kinase activity by the noncatalytic beta and gamma subunits. J Biol Chem 1996;271:17798-803.

20. Kramer HF, Witczak CA, Taylor EB, Fujii N, Hirshman MF, Goodyear LJ. AS160 regulates insulin- and contraction-stimulated glucose uptake in mouse skeletal muscle. J Biol Chem 2006;281:31478-85.

21. Frøsig C, Pehmøller C, Birk JB, Richter EA, Wojtaszewski JF. Exercise-induced TBC1D1 Ser237 phosphorylation and 14-33 protein binding capacity in human skeletal muscle. J Physiol 2010;588(Pt 22):4539-48.

22. Kramer HF, Witczak CA, Fujii N, Jessen N, Taylor EB, Arnolds
DE, et al. Distinct signals regulate AS160 phosphorylation in response to insulin, AICAR, and contraction in mouse skeletal muscle. Diabetes 2006;55:2067-76.

23. An D, Toyoda T, Taylor EB, Yu H, Fujii N, Hirshman MF, et al. TBC1D1 regulates insulin- and contraction-induced glucose transport in mouse skeletal muscle. Diabetes 2010;59: 1358-65.

24. Montgomery MK, Turner N. Mitochondrial dysfunction and insulin resistance: an update. Endocr Connect 2015;4:R1-15.

25. Ng JM, Azuma K, Kelley C, Pencek R, Radikova Z, Laymon C, et al. PET imaging reveals distinctive roles for different regional adipose tissue depots in systemic glucose metabolism in nonobese humans. Am J Physiol Endocrinol Metab 2012; 303:E1134-41.

26. Turner N, Cooney GJ, Kraegen EW, Bruce CR. Fatty acid metabolism, energy expenditure and insulin resistance in muscle. J Endocrinol 2014;220:T61-79.

27. Albers PH, Pedersen AJ, Birk JB, Kristensen DE, Vind BF, Baba $\mathrm{O}$, et al. Human muscle fiber type-specific insulin signaling: impact of obesity and type 2 diabetes. Diabetes 2015;64:48597.

28. Nyholm B, Qu Z, Kaal A, Pedersen SB, Gravholt CH, Andersen JL, et al. Evidence of an increased number of type IIb muscle fibers in insulin-resistant first-degree relatives of patients with NIDDM. Diabetes 1997;46:1822-8.

29. Stuart CA, McCurry MP, Marino A, South MA, Howell ME, Layne AS, et al. Slow-twitch fiber proportion in skeletal muscle correlates with insulin responsiveness. J Clin Endocrinol Metab 2013;98:2027-36.

30. Yan Z, Okutsu M, Akhtar YN, Lira VA. Regulation of exerciseinduced fiber type transformation, mitochondrial biogenesis, and angiogenesis in skeletal muscle. J Appl Physiol (1985) 2011; 110:264-74.

31. Picard M, Hepple RT, Burelle Y. Mitochondrial functional specialization in glycolytic and oxidative muscle fibers: tailoring the organelle for optimal function. Am J Physiol Cell Physiol 2012;302:C629-41.

32. Joseph AM, Hood DA. Relationships between exercise, mitochondrial biogenesis and type 2 diabetes. Med Sport Sci 2014; 60:48-61. 
33. Scarpulla RC, Vega RB, Kelly DP. Transcriptional integration of mitochondrial biogenesis. Trends Endocrinol Metab 2012; 23:459-66.

34. Bonen A. PGC-1alpha-induced improvements in skeletal muscle metabolism and insulin sensitivity. Appl Physiol Nutr Metab 2009;34:307-14.

35. Alston CL, Rocha MC, Lax NZ, Turnbull DM, Taylor RW. The genetics and pathology of mitochondrial disease. J Pathol 2017;241:236-50.

36. Collu-Marchese M, Shuen M, Pauly M, Saleem A, Hood DA. The regulation of mitochondrial transcription factor A (Tfam) expression during skeletal muscle cell differentiation. Biosci Rep 2015;35:e00221.

37. Kelley DE, Goodpaster B, Wing RR, Simoneau JA. Skeletal muscle fatty acid metabolism in association with insulin resistance, obesity, and weight loss. Am J Physiol 1999;277(6 Pt 1): E1130-41.

38. Sreekumar R, Nair KS. Skeletal muscle mitochondrial dysfunction \& diabetes. Indian J Med Res 2007;125:399-410.

39. Kim JY, Hickner RC, Cortright RL, Dohm GL, Houmard JA. Lipid oxidation is reduced in obese human skeletal muscle. Am J Physiol Endocrinol Metab 2000;279:E1039-44.

40. Gaster M, Rustan AC, Aas V, Beck-Nielsen H. Reduced lipid oxidation in skeletal muscle from type 2 diabetic subjects may be of genetic origin: evidence from cultured myotubes. Diabetes 2004;53:542-8.

41. Summermatter S, Shui G, Maag D, Santos G, Wenk MR, Handschin C. PGC-1 $a$ improves glucose homeostasis in skeletal muscle in an activity-dependent manner. Diabetes 2013;62:8595.

42. Wai T, Langer T. Mitochondrial dynamics and metabolic regulation. Trends Endocrinol Metab 2016;27:105-17.

43. Ni HM, Williams JA, Ding WX. Mitochondrial dynamics and mitochondrial quality control. Redox Biol 2015;4:6-13.

44. Anesti V, Scorrano L. The relationship between mitochondrial shape and function and the cytoskeleton. Biochim Biophys Acta 2006;1757:692-9.

45. Smirnova E, Griparic L, Shurland DL, van der Bliek AM. Dynamin-related protein Drp1 is required for mitochondrial division in mammalian cells. Mol Biol Cell 2001;12:2245-56.
46.Züchner S, Mersiyanova IV, Muglia M, Bissar-Tadmouri N, Rochelle J, Dadali EL, et al. Mutations in the mitochondrial GTPase mitofusin 2 cause Charcot-Marie-Tooth neuropathy type 2A. Nat Genet 2004;36:449-51.

47. Waterham HR, Koster J, van Roermund CW, Mooyer PA, Wanders RJ, Leonard JV. A lethal defect of mitochondrial and peroxisomal fission. N Engl J Med 2007;356:1736-41.

48. Bach D, Naon D, Pich S, Soriano FX, Vega N, Rieusset J, et al. Expression of Mfn2, the Charcot-Marie-Tooth neuropathy type 2A gene, in human skeletal muscle: effects of type 2 diabetes, obesity, weight loss, and the regulatory role of tumor necrosis factor alpha and interleukin-6. Diabetes 2005;54:2685-93.

49. Joseph AM, Adhihetty PJ, Buford TW, Wohlgemuth SE, Lees HA, Nguyen LM, et al. The impact of aging on mitochondrial function and biogenesis pathways in skeletal muscle of sedentary high- and low-functioning elderly individuals. Aging Cell 2012;11:801-9.

50. Chen H, Chomyn A, Chan DC. Disruption of fusion results in mitochondrial heterogeneity and dysfunction. J Biol Chem 2005;280:26185-92.

51. Pich S, Bach D, Briones P, Liesa M, Camps M, Testar X, et al. The Charcot-Marie-Tooth type 2A gene product, Mfn2, upregulates fuel oxidation through expression of OXPHOS system. Hum Mol Genet 2005;14:1405-15.

52. Zhang Z, Wakabayashi N, Wakabayashi J, Tamura Y, Song WJ, Sereda S, et al. The dynamin-related GTPase Opa1 is required for glucose-stimulated ATP production in pancreatic beta cells. Mol Biol Cell 2011;22:2235-45.

53. Boushel R, Gnaiger E, Schjerling P, Skovbro M, Kraunsøe R, Dela F. Patients with type 2 diabetes have normal mitochondrial function in skeletal muscle. Diabetologia 2007;50:790-6.

54. Rabøl R, Højberg PM, Almdal T, Boushel R, Haugaard SB, Madsbad S, et al. Effect of hyperglycemia on mitochondrial respiration in type 2 diabetes. J Clin Endocrinol Metab 2009; 94:1372-8.

55. Perry CG, Lally J, Holloway GP, Heigenhauser GJ, Bonen A, Spriet LL. Repeated transient mRNA bursts precede increases in transcriptional and mitochondrial proteins during training in human skeletal muscle. J Physiol 2010;588(Pt 23):4795810. 
56. Cartoni R, Léger B, Hock MB, Praz M, Crettenand A, Pich S, et al. Mitofusins $1 / 2$ and ERRalpha expression are increased in human skeletal muscle after physical exercise. J Physiol 2005; 567(Pt 1):349-58.

57. Ding H, Jiang N, Liu H, Liu X, Liu D, Zhao F, et al. Response of mitochondrial fusion and fission protein gene expression to exercise in rat skeletal muscle. Biochim Biophys Acta 2010; 1800:250-6.

58. Yan Z, Lira VA, Greene NP. Exercise training-induced regulation of mitochondrial quality. Exerc Sport Sci Rev 2012;40: 159-64. 